\title{
Repeated dispensing of codeine is associated with high consumption of benzodiazepines
}

\author{
Liliana C. Bachs ${ }^{1}$, Jørgen G. Bramness ${ }^{2}$, Anders Engeland ${ }^{2,3}$ and Svetlana Skurtveit ${ }^{2,4}$ \\ 1) Division of Forensic Toxicology and Drug Abuse, Norwegian Institute of Public Health \\ 2) Division of Epidemiology, Norwegian Institute of Public Health \\ 3) Department of Public Health and Primary Health Care, University of Bergen, Bergen, Norway \\ 4) Department of Pharmacy, University of Tromsø, Tromsø, Norway \\ Correspondence: Liliana Bachs, Norwegian Institute of Public Health, P.O. Box 4404 Nydalen, 0403 Oslo, Norway \\ E-mail: liliana.bachs@fhi.no Telephone:+47 23407892 Telefax:+47 23407878
}

\begin{abstract}
Aims: Our objective was to explore the use of codeine analgesics in individual patients in Norway, giving special attention to the $10 \%$ who consume the highest amounts.

Methods: We retrieved data from the Norwegian Prescription Database on patients who were dispensed at least one codeine analgesic prescription during 2006. We looked at age and gender specific 1-year periodic prevalence. The $10 \%$ of codeine users who were prescribed the highest amounts of the drug that year were further characterized. Age and gender distribution and concurrent high consumption of other potential drugs of abuse were also considered.

Results: In the year 2006, a total of 386,836 individuals filled at least one prescription for codeine analgesics from Norwegian pharmacies, excluding cancer patients. The crude prevalence for the use of codeine analgesics was $7.3 \%$ and $9.3 \%$ of the male and female Norwegian population, respectively. Twelve percent of women and $9 \%$ of men who filled a codeine prescription received 120 defined daily doses (DDD) or more of codeine analgesics in 2006 (moderate to high consumers). Fifty percent of those patients $(21,759)$ were also dispensed large amounts of benzodiazepines or carisoprodol over the same period. In comparison, only ten percent of patients who received fewer than 120 DDD of codeine analgesics were dispensed large amounts of benzodiazepines or carisoprodol.

Conclusions: A high percentage of the Norwegian population used codeine analgesics. One-year prevalence use of codeine increased with age and was higher for women at all ages. Our study showed that codeine use was mainly sporadic, but that a relatively large sub-group of users were dispensed repeated prescriptions of the drug in combination with other potential drugs of abuse.
\end{abstract}

Key Words: Codeine analgesics, Norwegian Prescription Database, prevalence, dispensed quantity, concurrent use, high consumers, abuse

\section{BACKGROUND}

Codeine is an opiate used for the treatment of mild to moderate pain.

There are large variations between European countries in the quantity and type of opioids used $(1,2)$. Drug sale statistics show that Norway has a high consumption of both codeine and morphine, while the sales of tramadol are low compared to the other Nordic countries (3). These variations may be explained by differences in national treatment guidelines and drug reimbursement systems.

Fixed combinations of analgesics containing codeine and paracetamol (acetaminophen) are widely prescribed in Norway (4). The use of these analgesics is more common in Norway than in other European countries (1-3), and accounts for more than $99 \%$ of the overall codeine consumption in the country.

The number of users of a given drug cannot be calculated from traditional wholesale data. Therapies may be used intermittently, duration of treatment may differ and dosing may differ from the defined daily dose (DDD). Further, drug use will not be evenly distributed for age or gender in the population. These limitations to the use of wholesale statistics in pharmacoepidemiology may be especially true when applied to an opioid analgesic because of the wide range of possible therapeutic doses.

The Norwegian Prescription Database (NorPD) covers the whole of Norway. NorPD makes it possible to calculate prevalence and to trace the prescription history of individual users and describe drug use by epidemiological parameters (5).

Despite the high use of analgesics containing codeine in Norway, and despite the fact that codeine, as all opioids, has abuse potential, only a limited number of studies have analyzed the prescription of these drugs (6-9). Codeine consumption in Norway is highly skewed, with a small group of patients receiving large amounts of the drug. An earlier study has shown that 
$1 \%$ of codeine users were prescribed as much as $16 \%$ of the total amount of codeine dispensed (6). In pharmacoepidemiology, skewness of consumption has been used to identify drugs with abuse potential (10).

In this study, we wanted to explore the age and gender distribution and the prevalence of users of analgesics containing codeine in Norway. Our analysis further focused on prevalence and population characteristics of the $10 \%$ of users of codeine who were prescribed the highest amounts of the drug, referred to below as "moderate/high users". Specifically we considered the group of moderate/high users for possible concurrent use of large amounts of other potential drugs of abuse.

\section{MATERIAL AND METHODS}

\section{Source of data}

Data was drawn from NorPD. From $1^{\text {st }}$ January 2004, all pharmacies in Norway are obliged to submit data electronically to the Norwegian Institute of Public Health on all dispensed prescriptions. This is done on a monthly basis. NorPD contains information on all prescription drugs, reimbursed or not, which are dispensed at pharmacies to individual patients outside institutions. Each record should contain a unique person-identifier, which makes it possible to identify chronologically all prescriptions by the individual patient and by the prescribing physician. Prescription data is collected from pharmacies, and so only captures prescriptions that are actually dispensed. The reimbursement code is also recorded and may be used as a proxy for diagnosis.

All medicines in Norway are classified according to the ATC classification system (11). Products containing codeine are included both in ATC code N02AA59 and in R05DA04. All analgesics in ATC code N02AA59 contain paracetamol and codeine and the majority of these combinations on the Norwegian market are tablets containing $30 \mathrm{mg}$ codeine phosphate and $400 \mathrm{mg}$ or $500 \mathrm{mg}$ paracetamol per tablet. These compounds were the subject of our study. For the product containing $400 \mathrm{mg}$ paracetamol, a DDD will represent $120 \mathrm{mg}$ codeine and $1,600 \mathrm{mg}$ paracetamol. For the product containing $500 \mathrm{mg}$ paracetamol, a DDD will represent $90 \mathrm{mg}$ codeine and 1,500 mg paracetamol. In spite of this flaw in accuracy, DDD is a practical tool to describe the amount of codeine dispensed. We did not feel it was necessary to correct for this difference in codeine DDD definition for the two compounds, in part because the product containing $400 \mathrm{mg}$ paracetamol is dominant in the market, and in part because the difference is of little importance for this study. A plain codeine brand containing only $8-10 \mathrm{mg}$ codeine is classified in the ATC code R05DA04, but sale of this brand was not included in the study since it is primarily used as a cough suppressant and constitutes only $0.6 \%$ of total sales of codeine in Norway. Use of codeine analgesics by individuals staying in hospitals and nursing homes, or codeine compounds sold as supplies to physician offices, amounted to $8.3 \%$ of the total DDD sold in Norway and was not a part of this analysis.

\section{Study population}

We identified all users of codeine compounds recorded in the NorPD during 2006. Patients with incomplete person-identifiers represented $1.9 \%$ of the prescriptions of codeine compounds and were excluded from further analysis. Subjects who presented one or more codeine prescriptions but who had the reimbursement code for cancer were also excluded.

Moderate/high users of codeine analgesics were defined as the $10 \%$ of codeine users prescribed the highest amount of DDD in 2006. To explore possible concurrent use of other potential drugs of abuse, we identified individuals who filled prescriptions for benzodiazepines and carisoprodol (a muscle relaxant with recognized abuse potential, often used together with benzodiazepines) in 2006 (12). High consumers of benzodiazepines and carisoprodol were identified as those who received 100 DDD or more and 15 DDD or more in 2006, respectively, as previously defined by others (6).

The concurrent drugs we studied were benzodiazepine anxiolytics marketed in Norway (ATC N05BA): diazepam, oxazepam, alprazolam; benzodiazepine hypnotics (ATC N05CD): nitrazepam, flunitrazepam, midazolam; and the centrally acting muscle relaxant agent carisoprodol (ATC M03BA02).

\section{Statistical methods}

Age and gender specific 1-year periodic prevalence was estimated by dividing the number of patients who had filled at least one prescription for codeine compounds in 2006 by the total number of persons in Norway in this age group on January 2006 .

Age and gender distribution of the Norwegian population in January 2006 were obtained from Statistics Norway (13).

Analyses were done using SPSS 14.0 for Windows.

\section{RESUlts}

\section{Users of codeine analgesics}

In the year 2006, a total of 386,836 individuals with a valid person identifier filled at least one prescription for codeine compounds from Norwegian pharmacies, excluding cancer patients. Of these, $44 \%$ were males $(169,501)$, yielding a crude prevalence for the use of codeine analgesics of $7.3 \%$ and $9.3 \%$ of the male and female Norwegian population, respectively. The 1year prevalence for different age groups is shown in figure 1. Prevalence increased with age for both genders, and was around $20 \%$ higher for females than for males. 


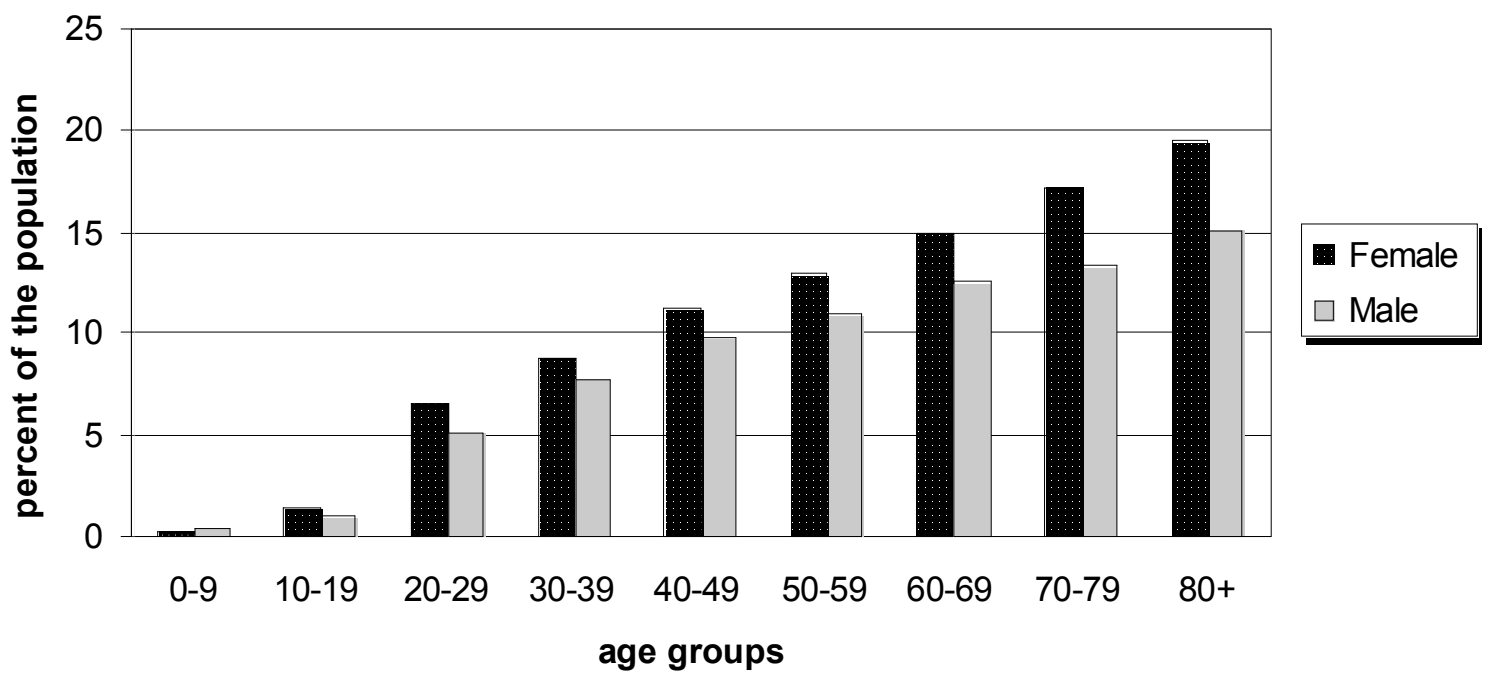

Figure 1. Gender and age specific 1 year periodic prevalence for individuals dispensed at least one codeine prescription in a Norwegian pharmacy in 2006.

The amount of drug received per patient varied widely (figure 2). Approximately $40 \%$ of the users received fewer than 12 DDD per year and $75 \%$ received fewer than 50 DDD per year. The $10 \%$ of users receiving the highest amounts of drug, received 120 DDD or more per year. Ten individuals were prescribed amounts over 2,000 DDD.

\section{Moderate/high users of codeine analgesics}

Moderate/high users of codeine analgesics were defined as the $10 \%$ of users who received the highest amounts of the drug in 2006, corresponding to 120 DDD or more. That would represent, for example, 3-4 tablets daily (the recommended dose for acute pain) for 4 months or more, or the use of 1 tablet or more a day throughout the year. Approximately $9 \%(15,225)$ of males and $12 \%(26,234)$ of females who filled a

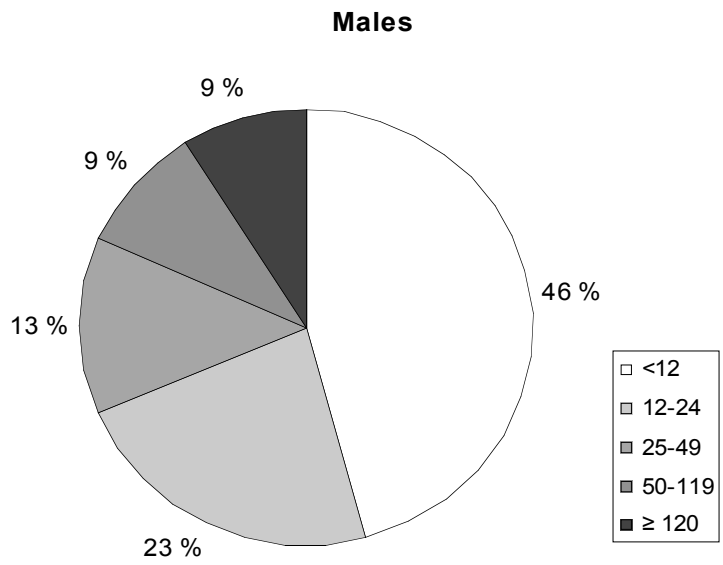

Figure 2. Amount of codeine prescribed to each patient in number of defined daily doses (DDD) prescribed that year, grouped into five categories. The pie chart shows the percentage of all codeine users in each group, for each gender. prescription for codeine that year received 120 DDD or more. The mean (median) age amongst these moderate/high users was 58 (57) years for males and 62 (61) years for females, while patients who received fewer than 120 DDD codeine that year were younger. Here, the mean (median) age was 50 (51) for males and 54 (54) for females. Age and gender distribution of moderate/high users is described in figure 3 .

Among moderate/high users of codeine, $42 \%$ had been prescribed high amounts of benzodiazepines or carisoprodol in the same period and $8 \%$ had been prescribed high amounts of both benzodiazepines and carisoprodol (figure 4). In contrast, only $10 \%$ of patients who received fewer than 120 DDD of codeine compounds were dispensed high amounts of benzodiazepines or carisoprodol or both. The percentage of codeine users prescribed high amounts of benzodiazepines, carisoprodol or both increased with the number of DDD of codeine prescribed up to 120 DDD (table 1).

Splitting DDD groups in the range above 120 DDD showed a similar age/gender distribution and a similar percentage of patients with concurrent high use benzodiazepines or carisoprodol as the group of 120 DDD and above as a whole.

The frequency of moderate/high consumers of codeine with concurrent high use of benzodiazepines and/or carisoprodol was in the range of $6.9-8.1 \%$ (females) and $4.0-5.7 \%$ (males) of all codeine users for the different age groups.

\section{DISCUSSION}

A high percentage of the Norwegian population $(\sim 8 \%)$ filled a prescription of codeine analgesics at least once during 2006. One-year prevalence use of codeine increased with age for both genders and was higher for women than for men at all ages above 10 years, rea- 


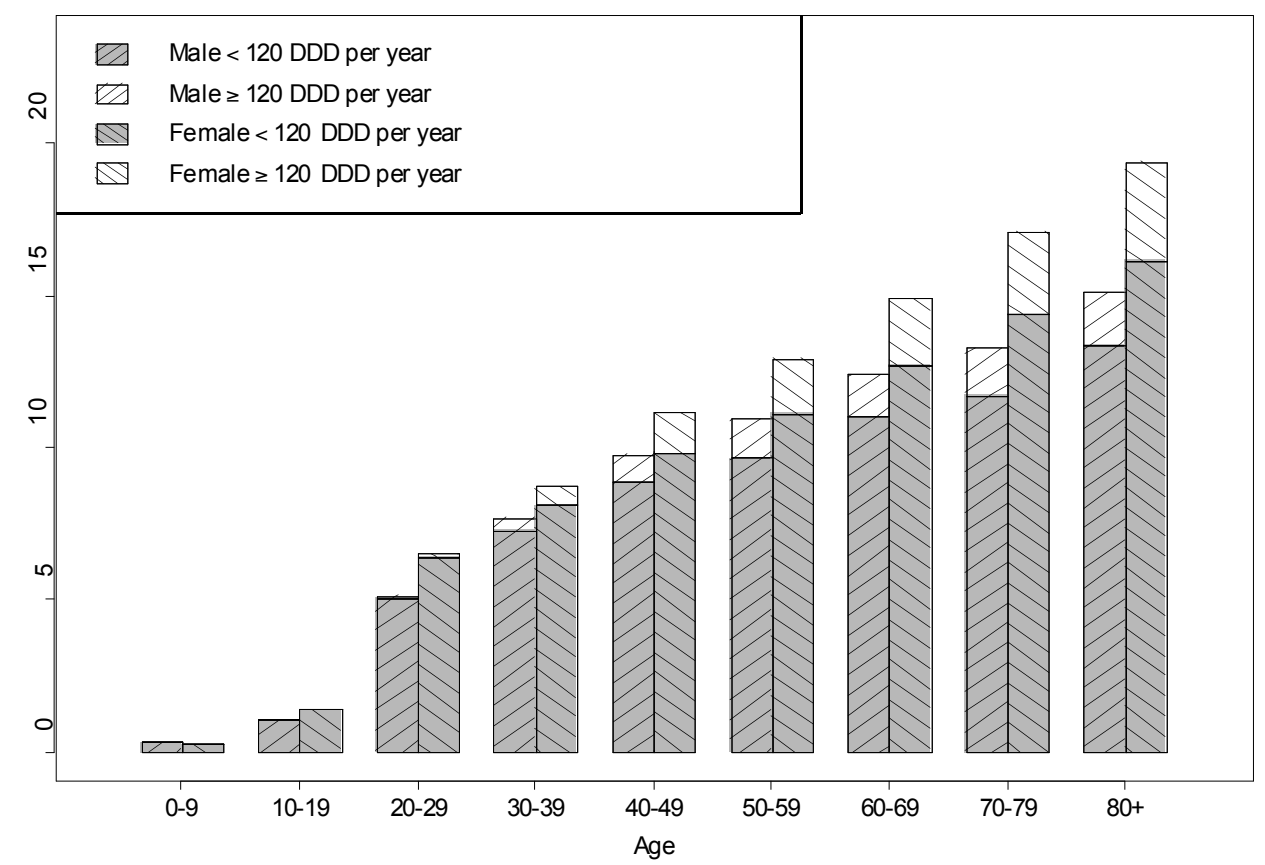

Figure 3. Age and gender distribution of patients dispensed codeine using under or above 120 defined daily doses (DDD) of codeine per year.

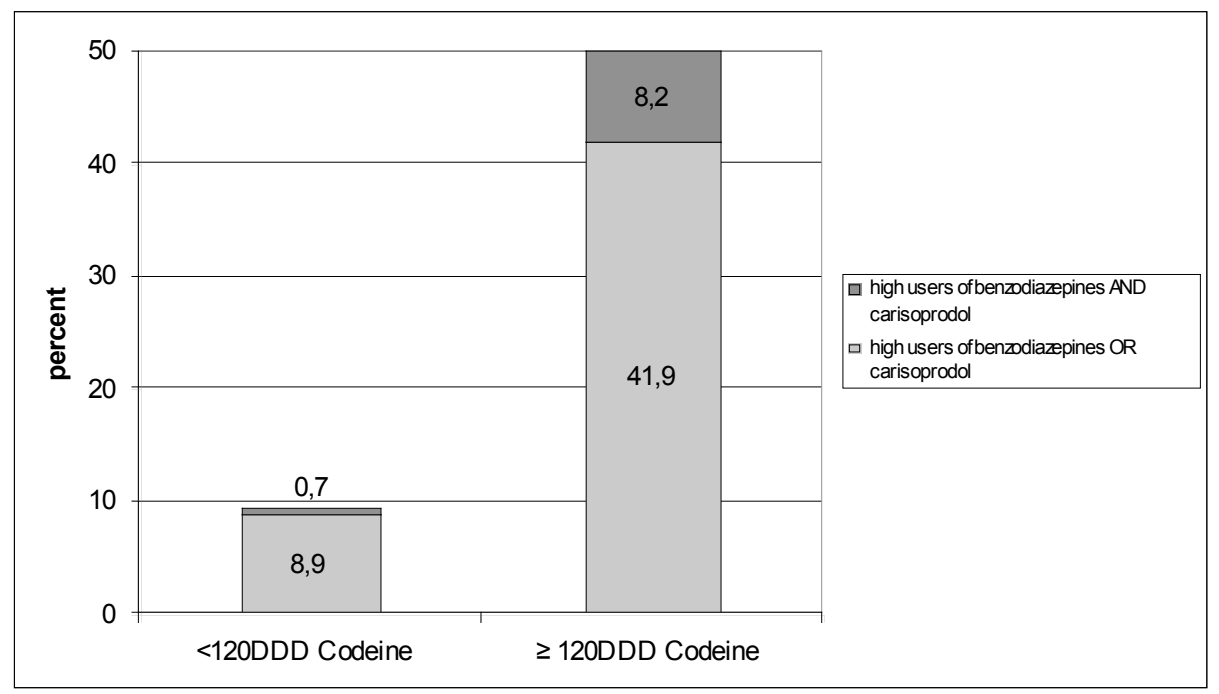

Figure 4. Concurrent use of $\geq 100$ defined daily doses (DDD) benzodiazepines and/or $\geq 15$ DDD carisoprodol among those who use less than 120 DDD or more of codeine analgesics respectively.

ching $20 \%$ for women older than 80 years. The $10 \%$ of users receiving the highest amount of the drugs in 2006 received 120 DDD or more. The prevalence of users receiving $120 \mathrm{DDD}$ or more of codeine in the general population increased with age to a maximum of 3.2 and 1.7 per hundred inhabitants for females and males over 80 years, respectively. Fifty percent of those patients $(21,759)$ were dispensed large amounts of benzodiazepines or carisoprodol over the same period. In comparison, $10 \%$ of patients who received fewer than 120 DDD of codeine analgesics were dispensed large amounts of benzodiazepines or carisoprodol.

The distribution of the amount of codeine prescribed to each individual per year was highly skewed. A previous study plotted a Lorenz curve for DDDs of codeine sold in 2004 and showed that $1 \%$ of codeine users were prescribed $16 \%$ of the total amount of codeine prescribed that year (6). While most users have a sporadic use of the drug, a substantial percentage of 
Table 1. Distribution of codeine users by amount of codeine received, plotted against concurrently dispensed amount of benzodiazepines and carisoprodol in the same year. Amounts in defined daily doses (DDD).

\begin{tabular}{|c|c|c|c|c|}
\cline { 2 - 5 } \multicolumn{1}{c|}{} & \multicolumn{4}{c|}{$\begin{array}{c}\text { Concurrent use of } \\
\text { benzodiazepines or carisoprodol N (\%) }\end{array}$} \\
\hline $\begin{array}{c}\text { DDD of codeine } \\
\text { analgesics in 2006 }\end{array}$ & $\mathrm{N}$ & $\begin{array}{c}\text { Amount of } \\
\text { benzodiazepines } \\
0-99 \text { DDD } \\
\text { and carisoprodol } \\
0-14 \text { DDD }\end{array}$ & $\begin{array}{c}\text { Amount of } \\
\text { benzodiazepines } \\
\geq 100 \text { DDD } \\
\text { or carisoprodol } \\
\geq 15 \text { DDD }\end{array}$ & $\begin{array}{c}\text { Amount of } \\
\text { benzodiazepines } \\
\geq 100 \text { DDD } \\
\text { and carisoprodol } \\
\geq 15 \text { DDD }\end{array}$ \\
\hline$<12$ & 1666353 & $158695(95.4 \%)$ & $7126(4.3 \%)$ & $532(0.3 \%)$ \\
\hline $12-24$ & 86115 & $78954(91.7 \%)$ & $6690(7.8 \%)$ & $471(0.5 \%)$ \\
\hline $25-49$ & 51688 & $44109(85.3 \%)$ & $7046(13.6 \%)$ & $533(1.0 \%)$ \\
\hline $50-119$ & 41221 & $30638(74.3 \%)$ & $9711(23.6 \%)$ & $872(2.1 \%)$ \\
\hline$\geq \mathbf{1 2 0}$ & $\mathbf{4 1 4 5 9}$ & $\mathbf{2 0 7 0 0 ( 4 9 . 9 \% )}$ & $\mathbf{1 7 3 7 9}(\mathbf{4 1 . 2 \% )}$ & $\mathbf{3 3 8 0}(\mathbf{8 . 2 \%})$ \\
\hline
\end{tabular}

users received high amounts. The highest amount we found was over 10 times the recommended daily dose every day of the year.

In this study, a moderate/high consumer was defined as a person who received 120 DDD or more during the year as this was the amount consumed by the $10 \%$ of patients with the highest consumption. A consumption of 120 DDD of codeine per year is not high in itself. It can represent, for example, the use of 3-4 tablets daily (the recommended dose for acute pain) for 4 months or more or the use of 1 tablet a day throughout the year. However, this amount does reflect repeated prescribing. Long term use of opioids for chronic non-malignant pain is not well documented. Norway's national guidelines advise a restrictive approach and careful assessment of pain relief versus unwanted effects in long term use (14). It is difficult to establish limits for "high" use of codeine analgesics as codeine compounds are largely prescribed to treat unspecified joint or muscle pain (15), which are not well defined diagnostically. Drug abuse is even more difficult to identify. A recent paper tried to identify pharmacoepidemiological parameters that can differentiate drugs of abuse from drugs without abuse potential. Skewness in the distribution of the drug prescribed to each individual per year was one of the signs that could differentiate drugs of abuse from other drugs without abuse potential (10). Prevalence of use and DDD distribution in our material was highly coincident with the results from a previous study of codeine prescriptions from a Norwegian county in 1994, suggesting a stable consumption profile over this time period.

Codeine may be associated with a lower addiction potential than, for example, morphine even though codeine use among multi-drug abusers is frequent $(16,17)$. Our study showed that codeine use was mainly sporadic, but that a relatively large sub-group of users received repeated dispensation of the drug.
Whether this represented opioid abuse or chronic therapeutic analgesic use was not possible to determine. The high concurrent use of other drugs with abuse potential, benzodiazepines and carisoprodol, in quantities above therapeutic recommendations among the moderate/high users of codeine can suggest the possibility of drug abuse. It is noteworthy that increasing consumption of codeine analgesics, even in the range below 120 DDD, is also associated with use of high amounts of benzodiazepines/carisoprodol, in our material. The percentage of patients with concurrent use of high amounts of benzodiazepines or carisoprodol did not further increase with dispensation of DDD above 120 DDD per year.

We acknowledge that complex and multi-symptomatic conditions in clinical practice may require drug use that does not fit therapeutic guidelines. Nevertheless, a subgroup of subjects who are receiving more than 365 DDD and up to several thousand DDD per year may be suggestive of drug abusers and is strongly suggestive of the diversion of codeine analgesics to the illegal market, as the personal ingestion of such large amounts of paracetamol would represent a high risk of liver damage.

\section{CONCLUSION}

A high percentage of the Norwegian population used codeine analgesics. One-year prevalence use of codeine increased with age and was higher for women at all ages. Ten percent of codeine users were prescribed 120 DDD or more in 2006. Approximately 50\% of those were concurrently prescribed high amounts of benzodiazepines and/or carisoprodol.

\section{CONFLICTS OF INTEREST}

None of the authors has any financial or personal conflict of interest that could influence their work in this study. 


\section{REFERENCES}

1. The Danish Medicine Agency, 2007. http://www.laegemiddelstyrelsen.dk/1024/visLSArtikel.asp?artikelID= 1780 .

2. De Conno F, Ripamonti C, Brunelli C. Opioid purchases and expenditure in nine western European countries: 'are we killing off morphine?' Palliat Med 2005; 19 (3): 179-184.

3. Medicines consumption in the Nordic Countries 1999-2003 [NOMESCO], 2007. http://www.nom-nos.dk/ nomersco.htm.

4. Rønning M, 2007. Drug Consumption in Norway 2002-2006. Folkehelseinstituttet, Legemiddelstatistikk.

5. Bramness JG, Hausken AM, Sakshaug S, Skurtveit S, Ronning M [Prescription of selective serotonin reuptake inhibitors 1990-2004]. Tidsskr Nor Laegeforen 2005; 125 (18): 2470-2473.

6. Bramness JG, Furu K, Engeland A, Skurtveit S. Carisoprodol use and abuse in Norway: a pharmacoepidemiological study. Br J Clin Pharmacol 2007; 64 (2): 210-218.

7. Eggen AE, Andrew M. Use of codeine analgesics in a general population. A Norwegian study of moderately strong analgesics. Eur J Clin Pharmacol 1994; 46 (6): 491-496.

8. Eggen AE. The use of controlled analgesics in a general population (15-59 years) - the influence of age, gender, morbidity, lifestyle and sociodemographic factors. Pharmacoepidemiol Drug Saf 1996; 5 (2): 101-111.

9. Straand J. Drug prescribing studies from general practice. Nor J Epidemiol 1999; 9 (2).

10. Hallas J, Stovring H. Templates for analysis of individual-level prescription data. Basic Clin Pharmacol Toxicol 2006; 98 (3): 260-265.

11. Lineamientos para la Clasificacion ATC y la asignación de DDD. WHO Collaborating Center for Drug Statistics Methodology, 2007.

12. Tobi H, Faber A, van den Berg PB, Drane JW, de Jong-van den Berg LT. Studying co-medication patterns: the impact of definitions 1. Pharmacoepidemiol Drug Saf 2007; 16 (4): 405-411.

13. Statistics Norway, 2007. http:/www.ssb.no.

14. Terapianbefaling: Bruk av opioider ved behandling av langvarige, non-maligne smertetilstander. 3. Statens legemiddelverk, 2002.

15. Rokstad K, Straand J, Fugelli P. General practitioners' drug prescribing practice and diagnoses for prescribing: the More \& Romsdal Prescription Study. J Clin Epidemiol 1997; 50 (4): 485-494.

16. Bachs L, Skurtveit S, Morland J. Codeine and clinical impairment in samples in which morphine is not detected. Eur J Clin Pharmacol 2003; 58 (12): 785-789.

17. Rowden AM, Lopez JR. Codeine addiction. DICP 1989; 23 (6): 475-477. 